\title{
A Fault Estimation Method Based on UIO Robust Residual Generators
}

\author{
LIU Yongjin ${ }^{1}$, FENG Gang ${ }^{1}$, ZHANG Lin ${ }^{1}$, WANG Wenfeng ${ }^{1,2,3}$, JIA Zhonghui ${ }^{1}$, ZHANG Bo $^{1, a}$ \\ ${ }^{1}$ Air Defense and Antimissile Institute, Air Force Engineering University, Xi'an, 710051, China \\ ${ }^{2}$ The 302ed Institute of the Tenth Academy of China Aerospace Science \& Industry Corporation; Guiyang 550009 , \\ China \\ ${ }^{3}$ School of Automation Science and Electrical Engineering, Beihang University, Beijing 100191, China
}

\begin{abstract}
This paper proposed a fault estimation method based on robust residual generators for a linear system. A system with additive actuator or component faults was considered in the case where the number of the independent faults was larger than that of the independent measurements. This method was achieved based on UIO robust residual generators and there was no need to design extra fault estimators. In this method, fault estimation was achieved via three steps. First, codingsets, which describe the sensitivity relationship between faults and generators, are designed. Second, a bank of robust residual generators are designed according to the coding sets. Finally, fault estimation is achieved by using the result of fault isolation and the output of robust residual generators. A sufficient condition on the application of the method was given and the asymptotic convergence property of the estimation error by using the method was proved. Simulation results demonstrate the effectiveness of the method.
\end{abstract}

\section{Introduction}

In recent years, with the rapid development of technologies, different kinds of systems are more complicated, and faults are easier to happen at the same time. Therefore, how to detect fault before it happens as soon as possible is the key to improve the reliability and safety of the system, it is also the hot topic that people research and there are series of research results [1-3]. The overall concept of fault diagnosis consists of fault detection, fault isolation, and fault analysis or identification. Fault diagnosis techniques are divided into model-based fault diagnosis techniques and data-driven design of fault diagnosis depending on the difference of character description and decision-making method between different systems. The former's core idea is to replace analytical redundancy with physical redundancy, and design observer to estimate the output of the system, then compare it with observed value to get information about fault [4-6].

As one of the model-based fault diagnosis techniques, the fault diagnosis technique based on robust residual generators is able to combine uncertainty of system and take full advantage of system's deep information, which is good for fault detection, fault identification, and fault isolation. The key of this method is to design a group of robust residual generators, and each robust residual generator is sensitive to a certain fault, but robust to the rest faults. Therefore, fault isolation can be achieved. Wang et al. designed robust observer for a linear system with additive actuator or component faults

\footnotetext{
a Corresponding author : dreamland_0628@163.com
} 
and isolated the fault [7]. Chen et al. designed robust residual generators based on UIO, then diagnosed and isolated component faults of a linear system[8]. The dimension of the fault diagnosis techniques based on UIO robust residual generators is same as the diagnosed system.

How to estimate fault is also the research emphasis in fault diagnosis. Hou, et al. designed UIO-based fault estimation method in the case where the number of the independent faults was larger than that of the independent measurements, and it also needs derivative of output, but derivative of output is hard to get[9]. In order to estimate actuator faults, Hamayun et al. designed sliding mode observer, but it also requires the number of the independent faults was larger than that of the independent measurements[10]. These two methods are both have limitations, and hard to meet requirements. Researches on the case where the number of the independent faults less than that of the independent measurements seem to be less[11].

When the number of the input channel independent faults(mainly include additive actuator and component faults) less than that of the independent measurements in linear system, in this paper, a fault estimation method based on UIO robust residual generators and suitable conditions were given, and achieved fault isolation and estimation.

\section{Problem Formulation}

Consider a typical linear system with additive actuator or component faults described as[12,13]

$$
\left\{\begin{array}{l}
\dot{x}(t)=A x(t)+B u(t)+D f(t) \\
y(t)=C x(t)
\end{array}\right.
$$

where, $x \in R^{n \times l}$ is the system state vector, $u \in R^{q \times l}$ is the system input vector, $y \in R^{p \times l}$ is the system output vector, $f \in R^{l \times 1}$ is the actuator or component fault signal. $A$ is system matric, $B$ is input matric, $C$ is output matric; $A, B, C$ are all with suitable dimensions and $(A, C)$ is observable. $D$ is fault direction vector, and $\operatorname{rank}(C)=p>1, \operatorname{rank}(D)>1$.

The fault diagnosis techniques based on UIO or sliding mode observer are able to achieve fault estimation in the case where $\operatorname{rank}(C D)=\operatorname{rank}(D)$, that is $l \leq p$; these two methods will not be able to directly estimate fault when the number of the input channel independent faults less than that of the independent measurements. Therefore, in this paper, a fault estimation method based on UIO robust residual generators will be designed after fault isolation.

\section{Fault Estimation}

Considering there are many researches on fault detection and fault isolation when $l>p$, the basic idea is to detect fault by designing Luenberger observers, and isolate faults by designing code sets and robust residual generators. The specific process is as follows.

\subsection{Fault Detection Strategy}

In order to detect fault, at first, design Luenberger observer for the linear system described as [1]

$$
\left\{\begin{array}{l}
\dot{\hat{x}(t)}=A \dot{x(t)}+B u(t)+L[y(t)-C \hat{x} \hat{(t)}] \\
r(t)=y(t)-C \hat{x}(t)
\end{array}\right.
$$

With an observer gain $L$ that ensures the observer stability.

Then design residual evaluation function $J(t)$ described as

$$
J(t)=\|r(t)\|
$$

The following inequalities are used to determine whether the fault occurs: 


$$
\begin{cases}J(t)>J_{t h}, & \text { Fault occurs } \\ J(t) \leq J_{t h}, & \text { Fault doesn't occurs }\end{cases}
$$

where $J_{\text {th }}$ is threshold value, which is small and random.

\subsection{Fault Isolation Strategy}

(1) Encode fault signal. Suppose $l=\{1,2, \ldots, l\}$ is the set composed of fault subscript, $m=\{1,2, \ldots, m\}$ is the set composed of residual subscript. $\Omega_{j} \subseteq m, j \in l$ is the fault coding set composed of robust residual generator subscripts which is sensitive to fault number $j, \Gamma_{i} \subseteq l, i \in m$ is the fault set composed of fault subscripts which is sensitive to robust residual generator number $i$, that is to say, it is the set composed of fault subscripts that influence robust residual generator number $i . \Omega_{j}$ and $\Gamma_{i}$ are both able to describe the corresponding relations between fault and residual, and achieve system fault isolation.

In order to isolate intercurrent fault as many as possible when $\operatorname{rank}(C)=p$, fault coding set can be designed to let robust residual generator be robust to $p$-1faults, sensitive to $l+1-p$ faults, so that $C_{l}^{p-1}$ robust residual generators can achieve $p$-1 intercurrent fault isolation.

(2) Design UIO-based robust residual generator

Suppose a typical linear system with additive unknown input are described as

$$
\left\{\begin{array}{l}
\dot{x}(t)=A x(t)+B u(t)+S \xi(t) \\
y(t)=C x(t)
\end{array}\right.
$$

where $S \zeta(t)$ is unknown input. And satisfies

$$
\left\{\begin{array}{l}
\operatorname{rank}(C S)=\text { rank } C \\
\left(C, A_{1}\right) \text { is observable }
\end{array}\right.
$$

where $A_{1}=A-S\left[(C S)^{T} C S\right]^{-1}(C S)^{T} C A$.

When the system described as (5) has fault, it can be rewritten as (1) which is described as a system with single input fault vector.

$$
\left\{\begin{array}{l}
\dot{x(t)}=A x(t)+B u(t)+S \xi(t) \\
y(t)=C x(t)
\end{array}\right.
$$

Proof. $I_{A}$ is component fault identification matrix which describes component faults, and $f^{1}(t)$ describes actuator faults, so that we can obtain

$$
\dot{x}(t)=A x(t)+B u(t)+I_{A} \Delta A_{n} x(t)+S \xi(t)+D^{1} f^{1}(t)
$$

By defining $D=\left[\begin{array}{lll}I_{A} & S & D^{1}\end{array}\right]$, the system described as(5) can be rewritten as

$$
\left\{\begin{array}{l}
\dot{x(t)}=A x(t)+B u(t)+D f(t) \\
y(t)=C x(t)
\end{array}\right.
$$

where fault vector $f(t)$ in the new state can be described as

$$
f(t)=\left[\begin{array}{c}
\Delta A_{n} x(t) \\
\xi(t) \\
f^{1}(t)
\end{array}\right]
$$

UIO-based robust residual generator i can be described as

$$
\left\{\begin{array}{l}
\dot{z}_{i}(t)=F_{i} z_{i}(t)+G_{i} u(t)+E_{i} y(t) \\
r_{i}(t)=M_{i} z(t)+H_{i} y(t)
\end{array}\right.
$$


where $i \in m, z_{i} \in R^{s \times 1}$ is the state vector of generator $i, \quad r_{i} \in R^{v \times 1}$ is residual output, $u \in R^{q \times 1}$ is the system input vector, $y \in R^{p \times 1}$ is the system output vector, $F_{i}, G_{i}, E_{i}, M_{i}, H_{i}$ are unknown matrices with suitable dimensions to be determined, $T_{i} \in R^{s \times n}$ is transformation matrix, and $T_{i}, F_{i}, E_{i}, M_{i}, H_{i}$ are determined by matric equation(12).

$$
\left\{\begin{array}{l}
E_{i}=E_{i 1}+E_{i 2} \\
T_{i} D^{(j)}=0, j \in l-\Gamma_{i} \\
T_{i} D^{(k)} \neq 0, k \in \Gamma_{i} \\
F_{i} T_{i}=T_{i} A-E_{i} C \\
E_{i 2}=F_{i} H \\
G_{i}=T_{i} B \\
M_{i} T_{i}+H_{i} C=0
\end{array}\right.
$$

where $F_{i}$ is Hurwitz matrix, $l-\Gamma_{i}$ consists of elements in the $l$ but not $\Gamma_{i}$.

In a similar way, fault detection of residual generator $i$ is given by

$$
J_{i}(t)=\left\|r_{i}(t)\right\|
$$

Design one-to-one fault indicator function

$$
R I_{i}= \begin{cases}1, & J_{i}(t)>J_{i}^{\text {th }}(t) \\ 0, & J_{i}(t) \leq J_{i}^{\text {th }}(t)\end{cases}
$$

where $R I_{i}$ is 1 or $0, J_{i}^{\text {th }}(t)$ is residual threshold value, which is small and random, and its value can be known through Monte Carlo experiment.

Suppose fault indicator function $F I_{j}$ of fault $\mathrm{j}$ described as

$$
F I_{j}=\bigcap_{k \in \Omega_{j}} R I_{k}, \quad j \in l
$$

$F I_{j}$ logical value is 1 or 0 , and it means

$$
\left\{\begin{array}{l}
F I_{j}=1, \quad \text { Fault joccurs } \\
F I_{j}=0, \quad \text { Fault j doesn't occur }
\end{array}\right.
$$

In this way, fault isolated can be achieved.

\subsection{Fault Estimation Strategy}

At presents, most of researches can only achieve fault detection and fault isolation when the number of the independent faults is larger than that of the independent measurements, researches on fault estimation seems to less. In this paper, UIO-based robust residual generator was designed at first to isolate fault when $l>p$, and then fault estimation method was designed after fault isolation. The specific process is as follows.

By defining

$$
\begin{aligned}
& Q_{i}=M_{i} F_{i}^{-1} T_{i} D\left(Q_{i} \in R^{v \times l}\right), Q=\left[Q_{1}^{T}, Q_{2}^{T}, \cdots, Q_{m}^{T}\right]\left(Q_{i} \in R^{(v m) \times l}\right) \\
& r(t)=\left[r_{1}^{T}(t), r_{2}^{T}(t), \cdots, r_{m}^{T}(t)\right]^{T}, \quad f(t)=\left[f^{(1)}(t), f^{(2)}(t), \cdots, f^{(l)}(t)\right]^{T}
\end{aligned}
$$

Fault estimation strategy is given by

$$
\hat{f(t)}=\{Q[F I(t)]\}^{+} r(t)
$$

where $F I(t)=\operatorname{diag}\left\{F I_{1}(t), F I_{2}(t), \cdots, F I_{l}(t)\right\}$ and $\{Q[F I(t)]\}^{+}$is pseudo-inverse matrix.

Theorem. If the system described as (1)holds: 


$$
\left\{\begin{array}{c}
\operatorname{rank}\left(C D^{\left(\Gamma_{i}\right)}\right)=\operatorname{rank}\left(D^{\left(\Gamma_{i}\right)}\right) \\
\operatorname{rank}\left(\begin{array}{cc}
s I_{n}-A & D^{\left(\Gamma_{i}\right)} \\
C & 0
\end{array}\right)=n+\operatorname{rank}\left(D^{\left(\Gamma_{i}\right)}\right) \\
\forall i \in m, \forall s \in \operatorname{Re}(s) \geq 0
\end{array}\right.
$$

where $I_{n}$ is identity matrix with $n$ dimension, $s$ is complex number with real part equal or greater than $0, \mathrm{D}^{(\Gamma i)}$ is matrix corresponding to $\Gamma_{i}$, we can achieve fault detection by (2)-(4) and fault isolation by(11)-(15). At the same time, asymptotically estimation of constant fault $\hat{f}(t)$ is given by

$$
\lim _{t \rightarrow \infty} \hat{f}(t)=f(t)
$$

Proof: It is easy to achieve fault detection and fault isolation by (2) (4)and(11) (15). We just prove that fault estimation is feasible.

By defining

$$
e_{i}(t)=z_{i}(t)-T_{i} x(t), \quad i \in m
$$

It can be inferred from (11)and (12) that

And

$$
\begin{aligned}
& \dot{e_{i}(t)=} \dot{z_{i}}(t)-T_{i} \dot{x}(t)=F_{i} z_{i}(t)+G_{i} u(t)+E_{i} y(t) \\
&-T_{i} A x(t)-T_{i} B u(t)-T_{i} D[F I(t)] f(t) \\
&=F_{i} e_{i}(t)-T_{i} D[F I(t)] f(t)
\end{aligned}
$$

$$
r_{i}=M_{i}\left[z_{i}(t)-T_{i} x(t)\right]
$$

Equation set (11) can be rewritten as

And we can obtain

$$
\left\{\begin{array}{l}
\dot{e}(t)=F_{i} e_{i}(t)-T_{i} D[F I(t)] f(t) \\
r_{i}(t)=M_{i} e_{i}(t)
\end{array}\right.
$$

$$
\begin{aligned}
\lim _{t \rightarrow \infty} r_{i}(t) & =\lim _{s \rightarrow 0} M_{i}\left(s I-F_{i}\right)^{-1} T_{i} D[F I(t)] f(t) \\
& =Q_{i}[F I(t)] f(t)
\end{aligned}
$$

It is because $r(t)=\left[r_{1}^{T}(t), r_{1}^{T}(t), \cdots, r_{m}^{T}(t)\right]^{T}$ that we obtain

$$
\lim _{t \rightarrow \infty} r(t)=Q[F I(t)] f(t)
$$

From equation set perspective, if we treat $\lim _{t \rightarrow \infty} r(t)$ as known quantity, $f(t)$ as matrix to be determined, (27) must have solution. Furthermore, there are $p$-1unknown quantities in (27)when $p$-1 faults occur, and depending on the designing rule, only one robust residual generator is robust to this $p$-1 faults in $\left\{r_{1}(t), r_{2}(t), \cdots, r_{m}(t)\right\}$, the rest of generators are all sensitive to this $p$ - 1 faults. For the minimum row of matrices in equation is 1 , the number of known quantities is $\left(C_{l}^{p-1}-1\right) \times 1$ at least in (25). Furthermore, $\left(C_{l}^{p-1}-1\right) \geq l-1>p-1,(27)$ has least square solution. The least square solution of (26) is the solution of equation based on the theory that equation must has solution. Therefore, we can obtain

$$
\hat{f(t)}=\{Q[F I(t)]\}^{+} r(t)
$$

\section{Simulation Results}

A linear system is employed with the following parameters:

$$
A=\left[\begin{array}{cccc}
-1 & -1 & -2 & 0 \\
0 & -1 & 1 & 2 \\
1 & 0 & -4 & 0 \\
0 & -1 & 0 & -1
\end{array}\right], C=\left[\begin{array}{llll}
1 & 0 & 1 & 0 \\
0 & 0 & 2 & 0 \\
0 & 1 & 0 & 1
\end{array}\right], B=D=\left[\begin{array}{llll}
1 & 0 & 1 & 1 \\
0 & 1 & 1 & 0 \\
0 & 1 & 0 & 1 \\
0 & 0 & 0 & 1
\end{array}\right],
$$




$$
u=\left[\begin{array}{llll}
1 & 1 & 1 & 1
\end{array}\right]^{T}, L=\left[\begin{array}{ccc}
0.9091 & -1.4545 & -0.6364 \\
-0.0909 & -0.5455 & 1.3636 \\
1 & -2 & 0 \\
-0.1818 & 0.0909 & -0.2727
\end{array}\right]
$$

There are four independent faults but three independent measurements in the system, so $C_{4}^{2}$ robust residual generators should be designed. Fault coding set is described as Table 1.

Table 1. Coding sets of simulation.

\begin{tabular}{|c|c|c|c|c|}
\hline & $f^{(1)}$ & $f^{(2)}$ & $f^{(3)}$ & $f^{(4)}$ \\
\hline$r_{1}$ & 0 & 0 & 1 & 1 \\
\hline$r_{2}$ & 0 & 1 & 0 & 1 \\
\hline$r_{3}$ & 0 & 1 & 1 & 0 \\
\hline$r_{4}$ & 1 & 0 & 0 & 1 \\
\hline$r_{5}$ & 1 & 0 & 1 & 0 \\
\hline$r_{6}$ & 1 & 1 & 0 & 0 \\
\hline
\end{tabular}

In the Table 1, "0"means that residual is robust to fault, while" 1 "means that residual is sensitive to fault. The parameters of UIO-based robust residual generators described as (11) are set as

$$
\begin{aligned}
& F_{1}=\left[\begin{array}{cccc}
-0.6875 & 1.0877 & -3.8540 & 0.0271 \\
1.2540 & -1.7675 & -3.3496 & 1.2325 \\
0.1279 & -0.2762 & -0.1769 & -0.2762 \\
0.1073 & 0.1391 & -0.5029 & -1.3609
\end{array}\right], F_{2}=\left[\begin{array}{cccc}
-6 & 0 & 0 & 0 \\
0 & 0 & 0 & 0 \\
1 & 0 & 0 & 0 \\
0 & 0 & 0 & 0
\end{array}\right], F_{3}=\left[\begin{array}{cccc}
-0.9952 & 1.7166 & -3.4606 & -0.0940 \\
0.1829 & -1.4208 & 1.5985 & 1.8899 \\
-0.0571 & -0.3318 & 0.0159 & -0.3318 \\
0.9362 & 0.0930 & -3.4584 & -1.4070
\end{array}\right], \\
& F_{4}=\left[\begin{array}{cccc}
-1.6667 & -0.6667 & 7 & 0.3333 \\
-1 & -1.5 & -3 & 0 \\
0 & 0 & -1 & 0 \\
0.6667 & -0.3333 & -0.5 & -1.8333
\end{array}\right], F_{5}=\left[\begin{array}{cccc}
-0.3554 & 1.6699 & -2.4903 & 0.1317 \\
2.5674 & -0.4912 & 1.4922 & 1.8942 \\
0.0007 & -0.3082 & -0.0320 & -0.3082 \\
-0.6567 & -0.1979 & -3.4815 & -1.6979
\end{array}\right], F_{6}=\left[\begin{array}{cccc}
-0.6348 & -0.6195 & 3.6172 & 0.5353 \\
-2.4375 & -1.5411 & 3.8105 & -0.0411 \\
0.1073 & 0.0980 & -1.4114 & 0.0980 \\
1.9089 & -0.3908 & -4.9477 & -1.8908
\end{array}\right],
\end{aligned}
$$

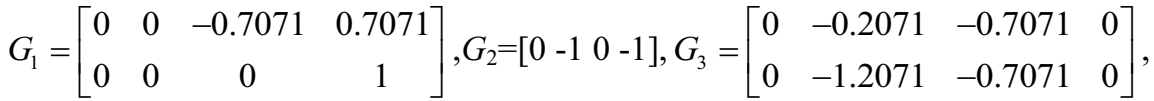

$$
\begin{aligned}
& G_{4}=\left[\begin{array}{llll}
-0.5 & 0 & 0 & -0.5
\end{array}\right], G_{5}=\left[\begin{array}{llll}
-0.5236 & 0 & -1.1236 & 0 \\
-0.5708 & 0 & -0.3708 & 0
\end{array}\right], G_{6}=\left[\begin{array}{llll}
-0.5774 & 0.5774 & 0 & 0
\end{array}\right], \\
& E_{1}=\left[\begin{array}{ccc}
0.7071 & -1.7015 & -0.6102 \\
0 & 0.0567 & 1.8271 \\
0 & -0.0452 & 0.1833 \\
0 & -0.2879 & -0.7816
\end{array}\right], E_{2}=\left[\begin{array}{ccc}
0.0635 & -1.0318 & 0.8939 \\
-0.7580 & 0.3790 & -1.7988 \\
0.3333 & 0.3333 & -0.3333 \\
0.9587 & -0.4794 & -1.4963
\end{array}\right], E_{3}=\left[\begin{array}{ccc}
0.5 & -0.8738 & -1.3350 \\
-0.5 & 1.4009 & 1.4161 \\
0 & -0.0687 & 0.1658 \\
0 & -0.4608 & -0.2411
\end{array}\right], \\
& E_{4}=\left[\begin{array}{ccc}
-0.8333 & 1.75 & 0.8333 \\
-0.5 & 0.25 & 1.25 \\
0 & 0 & 0 \\
0.3333 & -0.25 & -1.0833
\end{array}\right], E_{5}=\left[\begin{array}{ccc}
1.8907 & -1.5172 & -0.4999 \\
1.4347 & -0.0732 & 1.8994 \\
-0.1755 & 0.0568 & 0.1498 \\
-0.4568 & 0.1556 & -0.8761
\end{array}\right], E_{6}=\left[\begin{array}{ccc}
0.2109 & 0.7607 & 0.3665 \\
-1.4074 & 0.9537 & 2.1574 \\
0.0619 & -0.0310 & -0.0619 \\
1.1022 & -0.8011 & -1.8522
\end{array}\right] \text {, } \\
& M_{1}=\left[\begin{array}{cccc}
0.75 & -0.3535 & 0 & 0 \\
0 & 0 & 0 & 0 \\
-0.7071 & 0 & 0 & 0 \\
0.3536 & -0.5 & 0 & 0
\end{array}\right], M_{2}=\left[\begin{array}{llll}
1 & 0 & 0 & 0 \\
0 & 0 & 0 & 0 \\
1 & 0 & 0 & 0 \\
0 & 0 & 0 & 0
\end{array}\right] M_{3}=\left[\begin{array}{cccc}
0.4893 & -0.4893 & 0 & 0 \\
-0.1875 & 0.1875 & 0 & 0 \\
-0.25 & 0.25 & 0 & 0 \\
0.3018 & -0.3018 & 0 & 0
\end{array}\right] \text {, }
\end{aligned}
$$




$$
\begin{aligned}
& M_{4}=\left[\begin{array}{cccc}
1.5 & 0 & 0 & 0 \\
-0.5 & 0 & 0 & 0 \\
0.5 & 0 & 0 & 0 \\
-0.5 & 0 & 0 & 0
\end{array}\right], M_{5}=\left[\begin{array}{cccc}
1.3865 & 0.4214 & 0 & 0 \\
0.1888 & 0.3517 & 0 & 0 \\
-0.6 & 0.2 & 0 & 0 \\
0.3382 & -0.4854 & 0 & 0
\end{array}\right], M_{6}=\left[\begin{array}{cccc}
1.5773 & 0 & 0 & 0 \\
-0.5773 & 0 & 0 & 0 \\
0 & 0 & 0 & 0 \\
-0.5773 & 0 & 0 & 0
\end{array}\right] \\
& H_{1}=\left[\begin{array}{ccc}
1 & -0.8536 & 0.3535 \\
0 & 0 & 0 \\
0 & 0.5 & 0 \\
0 & 0 & 0.5
\end{array}\right], H_{2}=\left[\begin{array}{ccc}
1 & 0 & 0 \\
0 & 0 & 0.5 \\
0 & 0.5 & 0 \\
0 & 0 & 0.5
\end{array}\right], H_{3}=\left[\begin{array}{ccc}
1 & -0.75 & 0.6035 \\
0 & 0.25 & -0.1035 \\
0 & 0.5 & 0 \\
0 & 0 & 0.5
\end{array}\right] \text {, } \\
& H_{4}=\left[\begin{array}{ccc}
1.5 & -0.5 & -0.5 \\
0 & 0 & 0.5 \\
0 & 0.5 & 0 \\
0 & 0 & 0.5
\end{array}\right], H_{5}=\left[\begin{array}{ccc}
1.5236 & -1.0618 & 0.3382 \\
0.5708 & -0.1854 & 0.0146 \\
0 & 0.5 & 0 \\
0 & 0 & 0.5
\end{array}\right], H_{6}=\left[\begin{array}{ccc}
1.5774 & -0.7887 & -0.5774 \\
0 & 0 & 0.5 \\
0 & 0.5 & 0 \\
0 & 0 & 0.5
\end{array}\right] .
\end{aligned}
$$

It is easily to find that the dimension of the robust residual generators based on UIO is four, and it is same as the original system. By Monte Carlo experiment, threshold values are given by

$$
J_{t h}=0.1, J_{t h}^{1}=1 \times 10^{-8}, J_{t h}^{2}=5 \times 10^{-2}, J_{t h}^{3}=2 \times 10^{-3}, J_{t h}^{4}=5 \times 10^{-3}, J_{t h}^{5}=2 \times 10^{-3}, J_{t h}^{6}=2 \times 10^{-3} \text {. }
$$

And the parameters of fault estimation strategy described as(13) are set as

$$
\begin{aligned}
& T_{1}=\left[\begin{array}{cccc}
0 & -0.7071 & 0.7071 & 0 \\
0 & 0 & 0 & 1
\end{array}\right], T_{2}=\left[\begin{array}{llll}
0 & 0 & -1 & 0
\end{array}\right], T_{3}=\left[\begin{array}{cccc}
0 & -0.7071 & 0.5 & -0.5 \\
0 & 0.7071 & -0.5 & 0.5
\end{array}\right], \\
& T_{4}=[-0.5,0.5,-0.5,0.5], \quad T_{5}=\left[\begin{array}{cccc}
-0.5236 & -0.6 & 0.6 & -0.0764 \\
-0.5708 & 0.2 & -0.2 & 0.7708
\end{array}\right], T_{6}=\left[\begin{array}{lllll}
-0.5774 & 0.5774 & 0 & 0.5774
\end{array}\right] \text {, } \\
& Q_{1}=\left[\begin{array}{cccc}
0.2368 & 0 & 0.9676 & 0.6022 \\
0 & 0 & 0 & 0 \\
-0.2869 & 0 & -1.0860 & -0.6865 \\
0.0480 & 0 & 0.2824 & 0.1652
\end{array}\right], Q_{2}=\left[\begin{array}{cccc}
0.1622 & 0.1622 & 0 & 0 \\
0 & 0 & 0 & 0 \\
0.1622 & 0.1622 & 0 & 0 \\
0 & 0 & 0 & 0
\end{array}\right], \\
& Q_{3}=\left[\begin{array}{cccc}
0 & 0.0745 & 0.4345 & 0.1800 \\
0 & -0.0286 & -0.1665 & -0.0690 \\
0 & -0.0381 & -0.2220 & -0.0920 \\
0 & 0.0460 & 0.2680 & 0.1110
\end{array}\right], Q_{4}=\left[\begin{array}{cccc}
0.7071 & 0 & -1.4143 & -0.7071 \\
-0.2357 & 0 & 0.4714 & 0.2357 \\
0.2357 & 0 & -0.4714 & -0.2357 \\
-0.2357 & 0 & 0.4714 & 0.2357
\end{array}\right] \\
& , Q_{5}=\left[\begin{array}{cccc}
0 & 0 & -1.5652 & 0.0987 \\
0 & 0 & -0.6364 & -0.0579 \\
0 & 0 & 0.1296 & -0.1354 \\
0 & 0 & 0.4608 & 0.1667
\end{array}\right], Q_{6}=\left[\begin{array}{cccc}
0 & 0.8236 & 1.6472 & 0.8236 \\
0 & -0.3014 & -0.6029 & -0.3014 \\
0 & 0 & 0 & 0 \\
0 & -0.3014 & -0.6029 & -0.3014
\end{array}\right] .
\end{aligned}
$$

\section{Conclusion}

In this paper, we proposed a fault estimation method based on UIO robust residual fenerators for a linear system with input channel independent faults when the number of the independent faults was larger than that of the independent measurements. A sufficient condition on the application of the method and specific process of fault detection, fault isolation, fault estimation was given. At the same time, asymptotic convergence of residual estimation was proved. This method can achieve fault estimation only after we obtain the results of fault isolation by residual generators. The simulation results demonstrate that this proposed approach is efficient. 


\section{References}

1. Zhou D H, Hu Y Y. Fault diagnosis techniques for dynamic system. ActaAutomaticaSinica, 35(6): 748-758.(2009)

2. Ding S X. Model-based fault diagnosis techniques: design schemes, algorithms and tool. $2 \mathrm{nd}$. London: Springer, 1-9.(2013)

3. Sari A H A.Data-driven design of fault diagnosis systems: nonlinear multimode processes. Fachmedien Wiesbaden. NY: Springer, 11-30.(2014)

4. Qin L, He X, Zhou D H. A survey of fault diagnosis for swarm systems. Systems Science\&Control Engineering: An Open Acess Journal, 2(1):13-23.(2014)

5. Frank P M, Ding S X and Kppen-Seliger B. Current Developments in the Theory of FDI. Budapest, Hungary, 16-27.(2000)

6. Gentil S, Lesecq S, Barraud A. Improving decision making in fault detection and isolation using model validity. Engineering Applications of Artificial Intelligence, 22 (4-5): 534-545.(2009)

7. Wang X L, Wu P. Fault detection for control System based on unknown input observe. Lifting transportation machinery, (12):11-14.(2003)

8. Chen J, Patton R J. Robust model-based fault diagnosis for dynamic systems. US: Springer,65-108.(1999)

9. Hou M, Muller P C. Design of observers for linear systems with unknown inputs. Automatic Control, IEEE Transactions on, 37(6): 871- 875.(1992)

10. Hamayun M T, Edwards C, Alwi H. A fault tolerant control allocation scheme with output integral sliding modes. Automatica, 49(6): 1830-1837.(2013)

11. Zhu F. State estimation and unknown input reconstruction via both reduced-order and high-order sliding mode observers. Journal of Process Control, 22(1):296-302.(2012)

12. Zhang P. Design of Unknown Input Observer Based Fault Diagnosis Method for linear systems. Journal of ENGG College of Armed Pollce Force,24(6):29-32.(2008)

13. Hu Z K, Sun Y, Jiang B, He J, Zhang C F. An Optimal Unknown Input Observer Based Fault Diagnosis Method. ActaAutomaticaSinica,39(8):1225-1230.(2013) 